\title{
BIOANALYSIS THROUGH THE AGES
}

Dr Derek Stevenson, Division of Biochemical Sciences, University of Surrey, Guildford, UK

\section{Introduction and history}

This article highlights many of the issues of concern for analytical scientists working in the area of bioanalysis. It draws mainly on topics presented at the Reid International Bioanalytical Forum which is a microcosm of the issues faced by workers in this field and their attempts to solve them. The Forum was established in 1975 by the late Dr Eric Reid, Director of the Wolfson Bioanalytical Unit at the University of Surrey (see obituary in Bioanalysis (2010) 2(6), 1155). That unit was established with addressing the needs of industry and sharing expertise as part of its brief. The Forum has run every two years at the University since, the 2011 gathering being the $19^{\text {th }}$. Amongst other things this sharing of expertise amongst practitioners is always high on the agenda. It has of course evolved and some of that evolution is described here - hopefully not just as an historical account but also based on developments in the scientific aspects and the reasons such analysis is so widespread.

From 1975 until 1993 the Forum was run largely by Dr Reid firstly through the University and then through Guildford Academic Associates. There was an advisory board with a large input from the pharmaceutical industry especially to guide on the topics most relevant to that sector. From 1995 until 1999 the Forum was run by Dr Reid under the auspices of the Chromatographic Society. In 2001 Dr Reid took a back seat and the Forum has operated as The Forum Syndicate of the Chromatographic Society and was also named after its originator.

The proceedings of each meeting were published in a series of books "Methodological Surveys in Biochemistry" from the 1975 Forum until the 1997 Forum. The next three were published as a special issue in the journal Chromatographia. It is from these publications and the more recent programmes that most of the material here is taken. This author does not go back to the beginning, indeed the first Forum I attended was in 1979 (as a PhD student I would hasten to add) so comments on the early meetings are taken entirely from the proceedings.

There have been some consistent themes throughout the Bioanalytical Forum lifetime. Arguably the most valuable one was the encouragement to discuss the "rationale" for any particular method rather than just a recipe description. Linked to this was an encouragement to discuss approaches that had been tried unsuccessfully as well as the solutions. In the early days it was thought there was a lot of "folklore" in laboratories, or to put it another way lots of little things that might prevent you getting good results that might not be mentioned in mainstream scientific literature. It was basically undocumented but successful practices.

\section{Scope}

The term bioanalysis can mean different things to different people. A simple example would be measurement of drugs, metabolites and endogenous compounds in biological fluids. Another interest group might mean analysis of biologically active compounds, whether in biological samples or not. It could also include monitoring occupational and environmental exposure to chemicals by determining concentrations in biofluids, food, and environmental samples. The meetings have been dominated by 
presentations concerned with accurate measurement of drugs in biological fluids mainly plasma/serum, urine. This has been mainly as part of

phamacokinetic/bioavailablity studies necessary to develop new drugs and provide the data for regulatory authority approval. Other areas that occur are determination of endogenous compounds and biomarkers, environmental contaminants in animal tissues (such as dioxins), forensic toxicology where concentrations tend to be higher and therapeutic drug monitoring. One of the conferences did deliberately widen its footprint to embrace occupational/environmental samples. This particular meeting was centred on sample handling and preparation and so there was an opportunity to share expertise from seemingly different problem areas. The common problems included;-

- Measuring at low concentrations

- Lots of matrix components that might interfere

- Analytes that might not be stable

- Proving that your results were reliable

- Large numbers of samples

The clientele at the meetings have been dominated by the pharmaceutical industry and contract research organisations. There were plenty of academics either looking at new areas or techniques development/implementation but they were in the minority.

\section{Techniques}

It is interesting to note the techniques discussed in the first three issues of proceedings. HPLC, GC, TLC, luminescence, polarography and immunoassays were all reviewed initially along with sample preparation methods. In these early meetings there were twice as many contributions from analysts using $\mathrm{GC}$ as there were from HPLC proponents. Many of the methods involved extraction then derivatisation followed by GC. Even in those days mass spectrometry interfaced reasonably well with GC (unlike with HPLC) so MS was a common detector along with FID, ECD and AFID. In the context of HPLC methods using MS detection barely merited a mention.

There were occasional presentations concerning instrumental development for example sensors and biosensors, improvements in detectors such as fluorescence and electrochemical. As with many meetings manufacturers were keen to exhibit and to present their latest developments. Capillary electrophoresis first got a mention in the book from the 1989 Forum. It is still not common at the latest meeting, also true for supercritical fluid chromatography. As mentioned earlier many of the presenters were actual practitioners from the pharmaceutical industry and their suppliers particularly the contract research organisations that carried out much of their bioanalysis for them. By the time of the 1997 forum HPLC-MS methods were slightly more predominant than HPLC with other detectors. Since then HPLC-MS methods have dominated. It was originally thought by many, especially the inexperienced, as the solution to most methodological challenges. Of course its own folklore developed - the need to check for and overcome ion suppression problems to name but one.

Automation began to hit the radar in the third symposium and soon after that became common place as larger and faster throughput was required.

\section{Analytes}

In the early meetings the vast majority of discussions were about small molecular weight drugs and occasionally their metabolites. While there was still plenty of methodology for these types of compounds more recent meetings have shown an 
increase in analytes that are of larger molecular mass, peptides, polypeptides, oligonucleotides, proteins, antibodies etc. There has also been an increase in presentations for analysis of biomarkers reflecting the demand for the industry to provide increased proof of therapeutic benefit. For many years the Bioanalytical Forum had a major theme as well as encouraging presentations on case histories, validation etc. The themes often reflected a drug class particularly important at that time. Themes have included antipsychotic, peptides, anti-inflammatory, cardiovascular, anti infective, anti allergic, hypolipidaemic, peptide related drugs, anti asthmatics, mental alleviators, anti cancer drugs, sample preparation, metabolite isolation, enantiomers, molecular imprinting, the mass spec era and metabolic syndrome.

\section{Sample preparation/handling}

This was an area that invoked much attention in the early days. Bioanalysts needing to reach the low limits of detection required expended a lot of effort preparing samples. Liquid -liquid extraction and protein precipitation with organic solvent or strong acid were common. Although column chromatography and adsorption onto charcoal were also around solid phase extraction as we understand it today was in its infancy. Derivatization (because of the predominance of GC) and evaporation of solvents to dryness, was common. Despite the advances in instrumentation and the sensitivity now achievable for pure compound sample preparation is still necessary for most methods to be able to reach the detection levels required in biological samples. These of course have lowered which is why some of the expertise described in earlier books may be of use even in the mass spec era. Discussions about sources of error in bioanalytical methods have often centred on sample preparation.

\section{Quality of results}

Method validation, quality control, sample storage, use of internal standards and statistics all get a mention early on. What is clear is that there were few guidelines about validation and quality control/assurance. This has changed markedly and much time in more recent meetings has been devoted to dialogue with regulators and within the fraternity about ensuring reliable results. More details on the exact requirements have been forthcoming.

Great detail in how to conduct stability studies, method validation and monitoring method performance are available for example in the book from the 1997 forum. This is one area where the motivation of industrial Forum delegates diverges from much of academia. In that arena publication in a decent impact factor journal is a sufficient endpoint, which in the absence of quality assurance data substantiates very little.

\section{Conclusions}

So in the 1970s people worried about the sensitivity of their instruments, the reliability of their assays, how much quality control they needed, the stability of their samples. Hmm sounds familiar. Many of the issues at the early meetings remain issues now; the questions asked are more challenging often at lower concentration, with higher throughput required and with greater certainty about the results required. Better communication media such as e mail, internet, discussion boards etc. has not completely replaced some of the advantages of gathering in an informal environment to facilitate detailed discussion of solutions. 